Feldman R. \& Bakermans-Kranenburg M.J. (2017), Oxytocin: A parenting hormone, Current Opinion in Psychology, 15, 13-18.

DOI:10.1016/j.copsyc.2017.02.011

\title{
Oxytocin: A Parenting Hormone
}

Ruth Feldman ${ }^{1}$ \& Marian J. Bakermans-Kranenburg ${ }^{2,3}$

${ }^{1}$ Department of Psychology and the Gonda Brain Sciences Center, Bar-llan University, Ramat-Gan 52900, Israel

${ }^{2}$ Centre for Child and Family Studies, Leiden University, Leiden, Netherlands, ${ }^{3}$ Leiden Institute for Brain and Cognition (LIBC), Leiden University, Leiden, Netherlands

\section{Highlights}

- The expression of parenting in both human and non-human mammals is affected by hormones

- Oxytocin provides a neuroendocrine template for the effects of multiple hormones on parenting

- Oxytocin measured across pregnancy and the postpartum explains variance in parenting behavior

- Nasal administration of oxytocin influences neural responses to infant signals such as crying and laughter.

- Childhood experiences and attachment style may moderate the effects of oxytocin administration

\section{Key Words}

parenting, hormones, oxytocin, synchrony, intervention

\section{$\underline{\text { Acknowledgements }}$}

$\mathrm{RF}$ is supported by the Irving B. Harris Foundation, the Simms-Mann Foundation, and the German-Israel Foundation

MBK is supported by the European Research Council (ERC AdG) and the Gravitation program of the Dutch Ministry of Education, Culture, and Science and the Netherlands Organization for Scientific Research (NWO grant 024.001.003) 


\begin{abstract}
In non-human mammals mothering is hormone-dependent, with crucial roles for oxytocin and prolactin. While human parenting is not hormone-dependent, hormonal changes in oxytocin, vasopressin, prolactin, testosterone, and cortisol prime and accompany the expression of parenting. In the following we focus on oxytocin (OT) as a key hormone in human parenting. OT is a nine-amino-acid neuropeptide hormones which evolved from the ancient vasotocin molecule approximately 650 MYO. OT is implicated in sociality across vertebrate evolution and substantial research has pinpointed its role in birth, lactation, and maternal care in mammals. Over the last decade, studies have begun to examine peripheral levels of OT - in plasma, saliva, urine, and to lesser extend CSF - in humans as well as OT administration to parents. Correlational and experimental studies indicate that OT is associated with increased parent-child synchrony, sensitive parenting, and parental contact; interacts with other hormones, such as vasopressin, cortisol, or testosterone to create parent-specific effects; is associated with activation of key nodes in the parental brain, and is altered in conditions of high risk or parental psychopathology. We conclude by discussing the potential role of OT in interventions for high-risk parenting.
\end{abstract}




\section{Oxytocin: A Parenting Hormone}

Mammalian mothering in non-human animals is hormone-dependent. Hormonal changes during pregnancy and parturition causally determine the expression of maternal behavior and experimental manipulations on key hormones alter or eliminate maternal care [1-6]. Studies in rodents described the critical role of oxytocin (OT) and prolactin for the onset of maternal behavior; the contribution of stress hormones, particularly corticosterone, to maternal care [7-9]; and the role of vasopressin and testosterone particularly in the context of fatherhood [10-12]. In combination with sex-related hormones (estradiol, progesterone), these establish the neuroendocrine milieu that enables rodent mothers (and fathers in the $3-5 \%$ of mammalian species who are biparental) to parent [13-15].

Human parenting is not hormone-dependent; however, hormonal changes prime and accompany the expression of parenting [2, 16-18]. Humans' large associative cortex, neural plasticity, and massive limbic-cortical projections enable bottom-up behavior-based processing, so that committed parental care can trigger the hormones of parenting even without pregnancy and childbirth; for instance in primary-caregiving fathers or adoptive parents $[19,20]$. Parenting-related hormones are also linked with multiple neurobiological indices, such as brain activations, allelic variability, and autonomic functioning [21-25]. Evidence suggests that individual variations in OT are meaningfully associated with variations in maternal and paternal behavior and can index disruptions under high-risk conditions, such as premature birth, maternal depression, or contextual risk [1, 26, 27]. Maternal depression across the first years of life [28] has been linked with lower baseline OT [29] and attenuated OT response [30] in both mother and child. Moreover, "endocrine synchrony" between OT levels in parent and child have been demonstrated, highlighting the interactional transfer of parent-child neurobiology [31, 32]. Finally, human studies indicate that parental postpartum OT levels, in combination with parent-infant synchrony, are associated with later child well-being and social outcomes [33], underscoring their usefulness as early indices of adversity.

Overall, these studies highlight the importance of understanding the contribution of OT to human parenting and parent-child interaction.

Importantly, hormones other than OT meaningfully contribute to the expression of parental care. Vasopressin, prolactin, testosterone and cortisol have all been associated with various aspects of parenting in mothers and fathers; yet studies have also shown associations between OT and these hormones or their complex combined effects on parenting behavior. Here we present studies, particularly from the past decade, on OT and parenthood, guided by the perspective that the integrative oxytocinergic system provides a template for the integration of multiple hormones in support of human parenthood [34].

\section{Animal studies}

There are good reasons to submit that OT has been involved in life-sustaining and reproductive processes throughout animal evolutionary history [21], but rats, sheep, and voles dominate animal OT research. 
Nulliparous female rats avoid or attack pups, and show maternal behavior only after parturition, when progesterone levels go down, estradiol levels increase, and the OT production in the hypothalamic paraventricular nucleus and supraoptic nucleus is strongly upregulated. After OT injection, however, virgin females display all essential aspects of maternal behavior, including nest building and assuming a nursing posture $[35,36]$. Since olfactory stimuli appear to elicit negative behaviors to pups in virgin rats, OT (linked to the dopaminergic reward system) must play a role in the shift in the valence of pup odors from negative to positive [37].

Olfactory input does also play a role in concert with the oxytocinergic system in sheep. Similar to rats, sheep show maternal behavior only after parturition or, alternatively, with experimentally increased OT levels [38, 39]. A difference between sheep and rats is that postpartum female rats will care for any young, while a postpartum ewe rejects other lambs: she recognizes the specific odor of her own lamb, and develops an enduring and exclusive mother-infant bond with this specific lamb. When the main olfactory bulb is inactivated, ewes do care for any young, also those that are not her own, without forming a selective caregiving relationship.

Voles have become famous because they come in distinct types: Prairie voles are monogamous, nest together, and both parents take care of the young, whereas meadow voles and montane voles breed promiscuously, do not form pair bonds, and parental care is provided by females only. The voles are closely related genetically, and are similar in physical appearance. The difference is explained not by the density of OT receptors, but by different distributions of these receptors. Prairie voles - both males and females - have greater densities of OT receptors in the nucleus accumbens and the basolateral amygdala; montane voles have a greater density of OT receptors in the lateral septum. Similarly, densities of AVP differ between the vole types [37]. Equal levels of the peptides thus regulate behavior differently, illustrating the notion that the neurobiology of parenting is an intricate concerted action of many mechanisms, of which the salivary, plasma or even cerebrospinal fluid OT levels are only weak representations.

Even so, recent animal work has attracted attention for the very reason that increased OT levels in cerebrospinal fluid could be demonstrated after nasal OT administration. Doubts have been raised as to whether a sniff of OT does reach the brain [40, 41], and since taking samples from cerebral spinal fluid (CSF) in healthy human participants for the assessment of OT levels is too intrusive, the animal model is a welcome approach to address this issue [42]. In rats and mice increased brain and plasma OT levels after nasal application were found, both in the hippocampus and in the amygdala, with peak values between 30 and 60 min after administration [43]. Similarly, inhaling increased OT levels in CSF in rhesus monkeys [44]. Although levels in in CSF do not translate directly into levels in specific brain tissues, these studies add support to the hypothesis that the nasal anatomy offers several pathways for OT to reach the brain [45]. Behavioral effects attest to this idea, too, as inhaled OT increased positive social behaviors in newborn macaques [46].

\section{Studies in Humans}


Correlational designs. OT measured across pregnancy and the postpartum predicted the emergence of the human-specific maternal repertoire, indicating a priming effect [47]. In the first months of life, OT levels in caregiving fathers were no different from mothers and were associated with the parent-specific repertoire; affectionate touch, vocalizations, and gaze in mothers, and high arousal and stimulatory contact in fathers. Mothers who provided more affectionate contact with their infants exhibited OT increase, whereas for fathers it was not so much affectionate contact but high levels of stimulatory contact with their infant that resulted in an OT increase [48]. Moreover, infants as young as 4 months old displayed OT increase following synchronous interactions [31]. Longitudinal studies demonstrate the importance of parental OT functionality not only for the consolidation of children's long-term OT system but also for the development of social competencies. Parental OT combined with parent-infant synchrony predicted preschoolers' OT response and social reciprocity with best friends [33]. Similarly, in a 4-year follow-up parental brain response and OT in infancy predicted preschoolers' OT, emotion regulation, and socialization. Listening to mother's voice during stress elevated children's urinary OT and enabled better stress management [49]. OT levels also correlate with activation of key nodes in the parental brain when parents are exposed to their own infant stimuli [26]. These studies show parallels between OT functioning in humans and other mammals in the context of parenting and attest to the cross-generation transmission of OT in humans as mediated by parental behavior.

Experimental designs. In experimental research with intranasal OT administration two types of studies of parenting prevail: studies with actual parenting behavior as outcome, and studies focusing on behavioral or brain responses to infant stimuli in more controlled experimental designs.

The first two within-subject experiments with actual parenting behavior as outcome involved fathers, one group with typically developing toddlers and one group with toddlers in the autism spectrum. Fathers in both groups were significantly more vigorous and stimulating during play in the OT condition [50, 51]. This experimental finding mirrored and corroborated the correlational results that fathers who were more stimulating in their play had higher levels of salivary OT after the play session compared to fathers with less stimulating interactions [48]. In the third study, oxytocin administration increased fathers' touch and father-infant reciprocity [52]. Postnatally depressed mothers did however not benefit from experimentally elevated OT levels in terms of their sensitive interaction [53], nor did it ameliorate their depressive symptomatology, although it did improve their protective behavior toward their infants in the presence of an intrusive stranger [54].

In experimental designs with standardized infant stimuli, OT has been found to affect neural responses in meaningful ways. OT administration reduced amygdala activation and increased insula and inferior frontal gyrus activation in response to infant crying [55], which may prevent anxious or aversive feelings and enhance empathic concern. Moreover, when in a different sample context information accompanied the same infant crying sounds (now labeled as 'sick' or as 'bored'), OT enhanced insula, 
inferior frontal gyrus and amygdala responding to sick infant crying but lowered neural reactivity to the very same crying sounds when the infant was labeled as being bored [56]. Oxytocin thus appears to facilitate flexible adaptation of responses to infant crying depending on contextual information.

Importantly, OT may further facilitate parent-infant bonding by increasing the reward value of enjoyable infant signals. OT induced stronger functional connectivity between the amygdala and neural reward centers during exposure to infant laughter [57]. The up-regulation of the rewarding aspects of parenting after OT administration may also be indicated by an increase of activation in the right caudate when fathers viewed pictures of their own infant compared to an unknown infant [58].

Taken together, these studies seem to illustrate a supportive role of OT in the establishment of a secure parent-infant attachment. Unfortunately, however, a number of studies suggest that the positive effects on behavior or neurobiology may be minimal or absent in individuals with negative childhood experiences or insecure attachment styles [59-61]. For example, aggressive behavioral responses to infant crying decreased after OT administration compared to placebo, but only in participants without experiences of harsh parental discipline during childhood. For those who were disciplined harshly in the past no differences between the OT and placebo conditions emerged [62]. This limits the feasibility of OT as a stand-alone pharmacological treatment for those most in need of parenting support. The potential role of such treatment as an augmentative approach combined with supportive parenting intervention programs is in need of further exploration [63].

Figure 1: A research program for the role of oxytocin in parenting and development.

\section{Implications for future research and clinical practice}

Our brief review does not do justice to the vast literature on parenting and hormones in general and OT in particular. Yet, despite extant research, there is much we do not know about the neuroendocrine basis of human parenting, and we delineated a research program for the role of oxytocin in parenting and development (Figure 1). There is a need to develop reliable assays for biomarkers that likely play a key role in parenting, such as immune biomarkers, serotonin, or dopamine, preferably from saliva or non-invasive fractions. Longitudinal studies following children from birth and collecting hormones at numerous time-points from parent and child are still rare. The integration of multiple physiological systems, including genes, brain, hormones, autonomic functioning, and careful observations of behavior would provide new perspectives on the way OT and other hormones impact and are impacted by physiological and behavioral processes across development. 
Finally, there is a need to refine research on endocrine biomarkers of high-risk parenting. Hormones can provide early markers of disruptions to parenting processes, but much research is still needed to standardize measurements, define curves, and describe optimal versus non-optimal ranges at various ages and across cultural contexts. Further research is needed to fully understand how specific conditions, such as maternal depression, trauma, or personality disorder, translate into OT and other hormonal profiles, what is stable over time and what is transient, and how interventions can modify hormonal levels. Hormones provide a wealth of information on patterns of parental care across infant development in different cultural contexts and childrearing conditions and careful studies are needed to deepen our understanding of their direct and subtle impact on parenting. 


\section{References}

1. Feldman R: Oxytocin and social affiliation in humans. Horm behav 2012, 61 : 380-391.

2. ${ }^{* *}$ Feldman $\mathrm{R}$ : The neurobiology of mammalian parenting and the biosocial context of human caregiving. Horm behav 2016, 77:3-17.

This paper describes the role of OT in relation to other hormones involved in parenting

3. Lonstein JS, Lévy F, Fleming AS: Common and divergent psychobiological mechanisms underlying maternal behaviors in non-human and human mammals. Horm Behav 2016, 73:156-85.

4. Pryce CR: Socialization, hormones, and the regulation of maternal behavior in nonhuman simian primates. Adv Study Behav 1996, 25:423-473.

5. Rosenblatt JS: Psychobiology of maternal behavior: contribution to the clinical understanding of maternal behavior among humans. Acta Paediatr 1994, 397: 3-8.

6. Rosenblatt JS: Outline of the evolution of behavioral and nonbehavioral patterns of parental care among the vertebrates: Critical characteristics of mammalian and avian parental behavior. Scand J Psychol 2003, 44:265-271.

7. Brummelte S, Galea LA: Chronic corticosterone during pregnancy and postpartum affects maternal care, cell proliferation and depressive-like behavior in the dam. Horm behav 2010, 58:769-779.

8. Mann PE, Bridges RS: Lactogenic hormone regulation of maternal behavior. Prog Brain Res 2001, 133:251-262.

9. Pedersen CA, Prange AJ: Oxytocin and mothering behavior in the rat. Pharmacol Therapeutics 1985, 28:287-302.

10. *Carter CS: Oxytocin pathways and the evolution of human behavior. Ann rev psychol 2014, 65:17-39.

Describes the role of oxytocin (and vasopressin) in the expression of social behavior in humans and other mammals, including neurobiological, physiological, and behavioral correlates of oxytocin.

11. Wynne-Edwards KE: Hormonal changes in mammalian fathers. Horm Behav 2001, 40:139-145.

12. Rilling JK, Mascaro JS: The neurobiology of fatherhood. Curr Op in Psychol in press, this issue.

13. Bales KL: Parenting in animals. Curr Op in Psychol in press, this issue. 
14. Kleiman DG: Monogamy in mammals. Q Rev Biol 1977, 52:39-69.

15. Braun K, Champagne FA: Paternal influences on offspring development: behavioural and epigenetic pathways. J Neuroendocrinology 2014, 26:697706.

16. Gordon I, Zagoory-sharon O, Leckman JF, Feldman R: Oxytocin and the Development of Parenting in Humans. Biol psychiat 2010a, 68:377-382.

17. Gordon I, Zagoory-Sharon O, Leckman JF, Feldman R: Oxytocin, cortisol, and triadic family interactions. Physiol Behav 2010b, 101:679-684.

18. Galbally M, Lewis AJ, Van IJzendoorn MH, Permezel M: The role of oxytocin in mother-infant relations: a systematic review of human studies. Harv Rev Psychiatry 2011, 19:1-14.

19. Abraham E, Hendler T, Shapira-Lichter I, Kanat-Maymon Y, Zagoory-Sharon O, Feldman R: Father's brain is sensitive to childcare experiences. Proc Natl Acad Sci 2014, 111:9792-9797.

20. Bick J, Dozier M, Bernard K, Grasso D, Simons R: Foster mother-infant bonding: associations between foster mothers' oxytocin production, electrophysiological brain activity, feelings of commitment, and caregiving quality. Child dev 2013, 84:826-840.

21. ${ }^{* *}$ Feldman R, Monakhov M, Pratt M, Ebstein RP: Oxytocin pathway genes: evolutionary ancient system impacting on human affiliation, sociality, and psychopathology. Biol psychiat 2016, 79:174-184.

Provides an overview of the genetics and the evolution of the oxytocinergic system

22. Atzil S, Hendler T, Feldman R: Specifying the neurobiological basis of human attachment: brain, hormones, and behavior in synchronous and intrusive mothers. Neuropsychopharmacology 2011, 36:2603-2615.

23. Strathearn L, Fonagy P, Amico J, Montague PR: Adult attachment predicts maternal brain and oxytocin response to infant cues. Neuropsychopharmacology 2009, 34:2655-2666.

24. Swain JE, Ho SS: Neuroendocrine mechanisms for parental sensitivity: overview, recent advances and future directions. Curr Op in Psychol in press, this issue.

25. Parsons CE, Young KS, Stein A, Kringelbach ML: Intuitive parenting: understanding the neural mechanisms of parents' adaptive responses to infants. Curr Op in Psychol in press, this issue.

26. ${ }^{* *}$ Feldman R: The adaptive human parental brain: Implications for children's social development. Trends Neurosci 2015a, 38:387-399. 
Summarizes OT research in relation to the parental brain

27. Feldman R: Sensitive periods in human social development; New insights from research on oxytocin, synchrony, and high-risk parenting. Dev Psychopathol 2015b, 27:369-395.

28. Galbally M, Lewis AJ: Depression and Parenting: the need for improved intervention models. Curr Op Parenting, 2017.

29. Apter-Levi Y, Feldman M, Vakart A, Ebstein RP, Feldman R: Impact of maternal depression across the first six years of life on the child's mental health, social engagement, and empathy: The moderating role of oxytocin. $A m \mathrm{~J}$ Psychiat 2013, 170:1161-1168.

30. Pratt M, Apter-Levi Y, Vakart A, Feldman M, Fishman R, Feldman T, ZagoorySharon $O$, Feldman R: Maternal depression and child oxytocin response; moderation by maternal oxytocin and relational behavior. Depress anxiety 2015, 32:635-646.

31. Feldman R, Gordon I, Zagoory-Sharon O: The cross-generation transmission of oxytocin in humans. Horm Behav 2010, 58:669-676.

32. Feldman R, Singer M, Zagoory O: Touch attenuates infants' physiological reactivity to stress. Dev sci $2010,13: 271-278$.

33. Feldman R, Gordon I, Influs M, Gutbir T, Ebstein RP: Parental oxytocin and early caregiving jointly shape children's oxytocin response and social reciprocity. . Neuropsychopharmacology 2013, 38:1154-1162.

34. Feldman R: The neurobiology of human attachments. Trends Cog Sci 2017, 21:80-99.

35. Fahrbach SE, Morrell JI, Pfaff DW: Oxytocin induction of short-latency maternal behavior in nulliparous, estrogen-primed female rats. Horm behav 1984, 18:267-286.

36. Pedersen CA, Ascher JA, Monroe YL, Prange AJ: Oxytocin induces maternal behavior in virgin female rats. Science 1982, 216:648-650.

37. Numan M: Neurobiology of social behavior. London: Academic Press 2015.

38. Kendrick KM, Da Costa AP, Broad KD, Ohkura S, Guevara R, Lévy F, Keverne EB: Neural control of maternal behaviour and olfactory recognition of offspring. Brain res bull 1997, 44:383-395.

39. Keverne EB, Kendrick KM: Oxytocin facilitation of maternal behavior in sheep. Ann N Y Acad Sci 1992, 652:83-101.

40. Churchland PS, Winkielman P: Modulating social behavior with oxytocin: How does it work? What does it mean? Horm Behav 2012, 61:392-399. 
41. Leng G, Ludwig M: Intranasal oxytocin: Myths and delusions. Biol Psychiat 2016, 79:243-250.

42. Knop J, Van der Veen R, Joëls $M$ : The added value of rodent models in studying parental influences on offspring development: opportunitues, limitations and future perspectives. Curr Op in Psychol in press, this issue.

43. Neumann ID, Maloumby R, Beiderbeck DI, Lukas M, Landgraf R: Increased brain and plasma oxytocin after nasal and peripheral administration in rats and mice. Psychoneuroendocrinology 2013, 38:1985-1993.

44. Chang SW, Barter JW, Ebitz RB, Watson KK, Platt ML: Inhaled oxytocin amplifies both vicarious reinforcement and self reinforcement in rhesus macaques (macaca mulatta).

Proc Natl Acad Sci USA 2012, 109:959-964.

45. Quintana DS, Alvares GA, Hickie IB, Guastella AJ: Do delivery routes of intranasally administered oxytocin account for observed effects on social cognition and behavior? A two-level model. Neurosci Biobehav Rev 2015, 49:182-192.

46. Simpson EA, Sclafani V, Paukner A, Hamel AF, Novak MA, Meyer JS, Suomi SJ, Ferrari PF: Inhaled oxytocin increases positive social behaviors in newborn macaques.

Proc Natl Acad Sci 2014, 111:6922-6927.

47. Feldman R, Weller A, Zagoory-Sharon O, Levine A: Evidence for a neuroendocrinological foundation of human affiliation plasma oxytocin levels across pregnancy and the postpartum period predict mother-infant bonding. Psychol Sci 2007, 18:965-970.

48. Feldman R, Gordon I, Schneiderman I, Weisman O, Zagoory-Sharon O: Natural variations in maternal and paternal care are associated with systematic changes in oxytocin following parent-infant contact.

Psychoneuroendocrinology 2010, 35:1133-1141.

49. Seltzer LJ, Ziegler TE, Pollak SD: Social vocalizations can release oxytocin in humans. Proc R Soc Lond B Biol Sci 2010, 277:2661-2666.

50. **Naber F, Van IJzendoorn MH, Deschamps P, Van Engeland H, BakermansKranenburg MJ: Intranasal oxytocin increases fathers' observed responsiveness during play with their children: A double-blind withinsubject experiment. Psychoneuroendocrinology 2010, 35:1583-1586.

Intranasal administration of oxytocin promoted fathers' stimulatory play with their toddlers. This experimental study complements the correlational study (ref 43) that fathers who were more stimulating in their play had higher levels of salivary OT after the play session compared to fathers with less stimulating interactions. 
51. Naber FBA, Poslawsky IE, Van IJzendoorn MH, Van Engeland H, BakermansKranenburg MJ: Brief Report: Oxytocin Enhances Paternal Sensitivity to a Child with Autism: A Double-Blind Within-Subject Experiment with Intranasally Administered Oxytocin. J Autism Dev Disorders 2013, 43:224229.

52. Weisman O, Zagoory-Sharon O, Feldman R: Oxytocin administration to parent enhances infant physiological and behavioral readiness for social engagement. Biol Psychiat 2012, 72:982-989.

53. Mah BL, Van IJzendoorn MH, Out D, Smith R, Bakermans-Kranenburg MJ: The Effects of Intranasal Oxytocin Administration on Sensitive Caregiving in Mothers with Postnatal Depression. Child Psychiat \& Hum Dev 2016, Online First.

54. Mah BL, Bakermans-Kranenburg MJ, Van IJzendoorn MH, Smith R: Oxytocin promotes protective behavior in depressed mothers: a pilot study with the enthusiastic stranger paradigm. Depress Anxiety 2015, 32:76-81.

55. Riem MME, Bakermans-Kranenburg MJ, Pieper S, Tops M, Boksem MAS, Vermeiren RRJM, Van IJzendoorn MH, Rombouts SARB: Oxytocin Modulates Amygdala, Insula, and Inferior Frontal Gyrus Responses to Infant Crying: A Randomized Controlled Trial. Biol Psychiat 2011, 70:291-297.

56. ${ }^{* *}$ Riem MME, Voorthuis A, Bakermans-Kranenburg MJ, Van IJzendoorn MH: Pity or peanuts? Oxytocin induces different neural responses to the same infant crying labeled as sick or bored. Dev Sci 2014, 17:248-256.

Oxytocin significantly increased insula and inferior frontal gyrus responding to crying labeled as coming from a sick infant, but decreased activation in these brain regions in response to the same crying labeled as coming from a bored infant. The study thus demonstrates that the crying stimulus itself is less important than the subject's perception of the context of the cry, and that this perception is affected by oxytocin, promoting flexible adaptation of the caregiving response.

57. Riem MME, Van IJzendoorn MH, Tops M, Boksem MAS, Rombouts SARB, Bakermans-Kranenburg MJ: No Laughing Matter: Intranasal Oxytocin Administration Changes Functional Brain Connectivity during Exposure to Infant Laughter. Neuropsychopharmacology 2012, 37:1257-1266.

58. Wittfoth-Schardt D, Grunding J, Wittfoth $M$, Lanfermann $H$, Heinrichs $M$, Domes $\mathrm{G}$, Buchheim A, Gündel $\mathrm{H}$, Waller $\mathrm{C}$ : Oxytocin modulates neural reactivity to children's faces as a function of social salience. Neuropsychopharmacology 2012, 37:1799-1807.

59. Bakermans-Kranenburg MJ, Van IJzendoorn MH: Sniffing around oxytocin: review and meta-analyses of trials in healthy and clinical groups with implications for pharmacotherapy. Transl Psychiat 2013, 3:e258. 
60. Olff M, Frijling JL, Kubzansky LD, Bradley B, Ellenbogen MA, Cardoso C, Bartz $J A$, Yee JR, Van Zuiden M: The role of oxytocin in social bonding, stress regulation and mental health: An update on the moderating effects of context and interindividual differences. Psychoneuroendocrinology 2013, 38:1883-1894.

61. Shamay-Tsoory SG, Abu-Akel A: The Social Salience Hypothesis of Oxytocin. Biol Psychiat 2016, 79:194-202.

62. Bakermans-Kranenburg MJ, Van IJzendoorn MH, Riem MME, Tops M, Alink LRA: Oxytocin decreases handgrip force in reaction to infant crying in females without harsh parenting experiences. Soc Cog and Affect Neurosci 2012, 7:951-957.

63. Van IJzendoorn MH, Bakermans-Kranenburg MJ: The role of oxytocin in parenting and as augmentative pharmacotherapy: critical issues and bold conjectures. J Neuroendocrinology 2016, 28. 
Figure 1

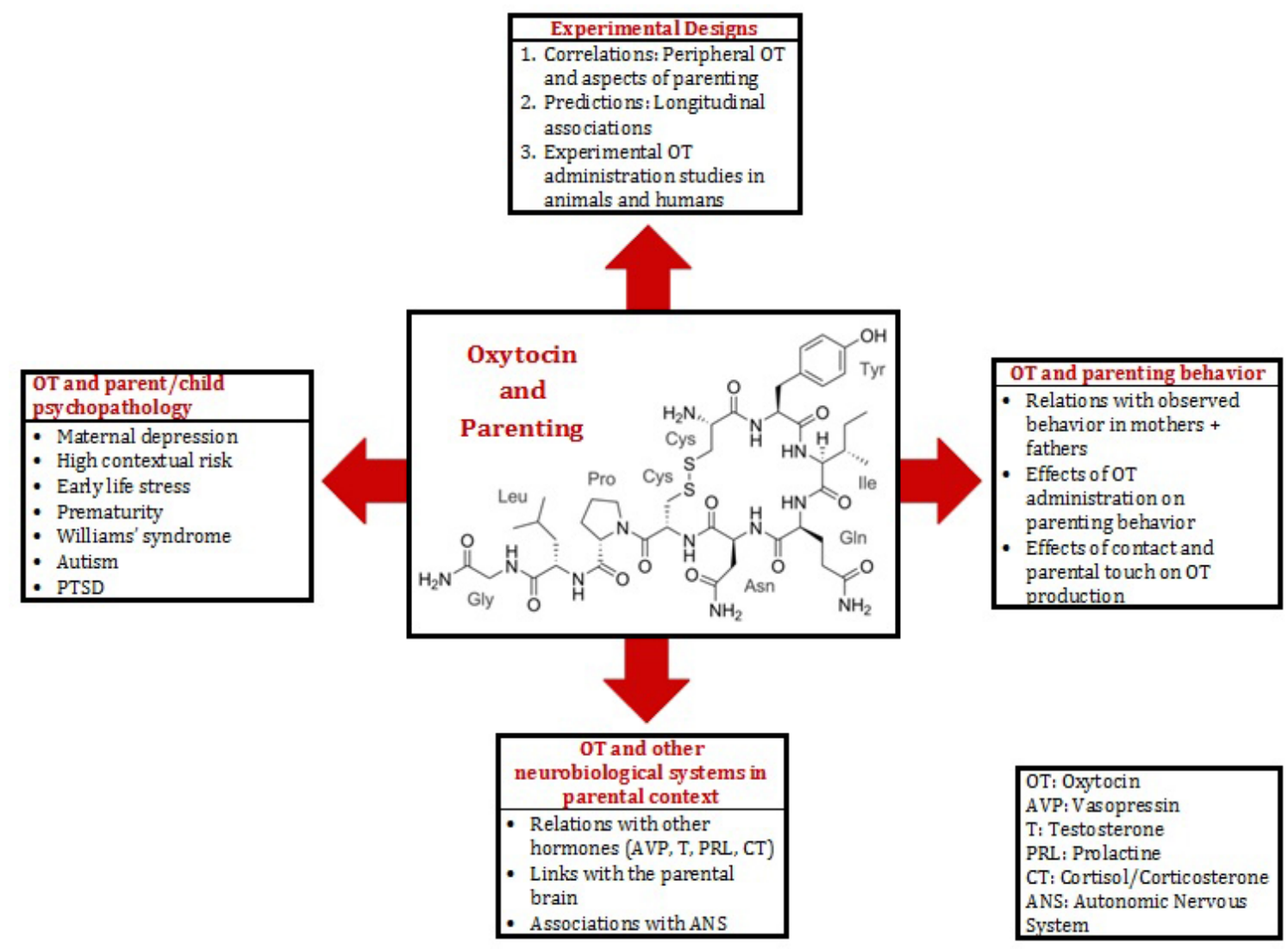

